\title{
Differential polyadenylation of ribosomal RNA during post-transcriptional processing in Leishmania
}

\author{
S. DECUYPERE ${ }^{1,2}$, J. VANDESOMPELE ${ }^{3}$, V. YARDLEY ${ }^{4}$, S. DE DONCKER ${ }^{1}$, \\ T. LAURENT ${ }^{1}$, S. RIJAL ${ }^{5}$, A. LLANOS-CUENTAS ${ }^{6}$, F. CHAPPUIS ${ }^{7}$, \\ J. AREVALO ${ }^{6}$ and J.-C. DUJARDIN ${ }^{1 *}$ \\ ${ }^{1}$ Department of Parasitology, Unit of Molecular Parasitology, Prince Leopold Institute of Tropical Medicine, \\ Antwerp B-2000, Belgium \\ ${ }^{2}$ Department of Biomedical Sciences, University of Antwerp, Antwerp B-2080, Belgium \\ ${ }^{3}$ Centre for Medical Genetics, Ghent University Hospital 1K5, Ghent B-9000, Belgium \\ ${ }^{4}$ Department of Infectious and Tropical Diseases, London School of Hygiene and Tropical Medicine, London WC1E7HT, UK \\ ${ }^{5}$ B.P. Koirala Institute of Health Sciences, Dharan, Nepal \\ ${ }^{6}$ Departamento de Bioquimica, Biologia Molecular y Farmacologia, Facultad de Ciencias y Filosofia and Instituto de Medicina \\ Tropical "Alexander von Humboldt", Universidad Peruana Cayetano Heredia, Lima 100, Peru \\ ${ }^{7}$ Department of Community Medicine, Travel and Migration Medicine Unit, Hopitaux Universitaires de Geneve, \\ Geneva, Switzerland
}

(Received 10 December 2004; revised 15 February 2005; accepted 28 February 2005)

\section{SUMMARY}

The protozoan parasite Leishmania belongs to the most ancient eukaryotic lineages and this is reflected in several distinctive biological features, such as eukaryotic polycistronic transcription and RNA trans-splicing. The disclosure of this organism's unusual characteristics leads to a better understanding of the origin and nature of fundamental biological processes in eukaryotes. Here we report another unusual phenomenon as we demonstrate that precursor ribosomal RNA can be extensively polyadenylated during post-transcriptional processing $\dagger$. Furthermore, we demonstrate that the degree of precursor rRNA polyadenylation is variable in different strains and in the different life-stages of a strain.

Key words: Leishmania, rRNA polyadenylation.

\section{INTRODUCTION}

Eukaryotic RNA can be subdivided into 2 classes, translated RNA which encodes proteins and nontranslated RNA which comprises rRNA, tRNA, snRNA and snoRNA.

The translated RNA or mRNA is transcribed by RNA polymerase II and is polyadenylated at the 3 ' end by poly(A) polymerase upon termination of transcription of the primary mRNA transcript. Poly(A)-tails are considered to be a distinctive feature of mRNA, as it seems that RNA polymerase II is required for efficient polyadenylation. The exact role of poly(A)-tails is still unclear but it has been proposed that the poly(A)-tail affects translation, stability, and nuclear export (Colgan and Manley, 1997; Hirose and Manley, 1998; Zhao, Hyman and Moore, 1999; Proudfoot and O'Sullivan, 2002).

The non-translated RNA consists mainly of ribosomal RNA (rRNA) which represents the vast

\footnotetext{
* Corresponding author: 'Prins Leopold' Instituut voor Tropische Geneeskunde, Unit of Molecular Parasitology, Nationalestraat 155, B-2000 Antwerpen, Belgium. Tel : + 32 3 2476358. Fax: + 323 2476359. E-mail: jcdujard@itg.be $\dagger$ Nucleotide sequence data reported in this paper are available in the GenBank database under Accession no. AY730681.
}

majority of the nucleic acid content of an organism (Sollner-Webb and Mougey, 1991). In most eukaryotes, the rRNA coding genes are tandemly repeated with each repeat encoding for $18 \mathrm{~S}, 5.8 \mathrm{~S}$ and $28 \mathrm{~S}$ rRNA. The whole repeat is transcribed by RNA polymerase I, producing a primary transcript that includes external transcribed spacers (ETS) and internal transcribed spacers (ITS). Further processing of this primary transcript by exo- and endonucleases leads to 3 mature rRNA transcripts: (i) $18 \mathrm{~S}$, part of the ribosome's small subunit (SSU) and (ii) $5.8 \mathrm{~S}$ and $28 \mathrm{~S}$, part of the ribosome's large subunit (LSU) (Reeder, 1990; Fromont-Racine et al. 2003). The LSU also contains 5S rRNA but the gene encoding this rRNA is usually not linked to the rRNA repeat and is transcribed by polymerase III (HernandezRivas et al. 1992; Paule and White, 2000).

The pathogenic Leishmania species, which belong to the order of Kinetoplastida, cause a diverse group of vector-borne diseases called leishmaniasis, that range from self-healing cutaneous lesions to fatal visceral disease. Members of the order Kinetoplastida diverged very early in eukaryotic evolution (Sogin et al. 1989) and this is clearly reflected in several aspects of their RNA metabolism. mRNA is transcribed as polycistronic primary transcripts as in prokaryotes, and subsequently processed to 


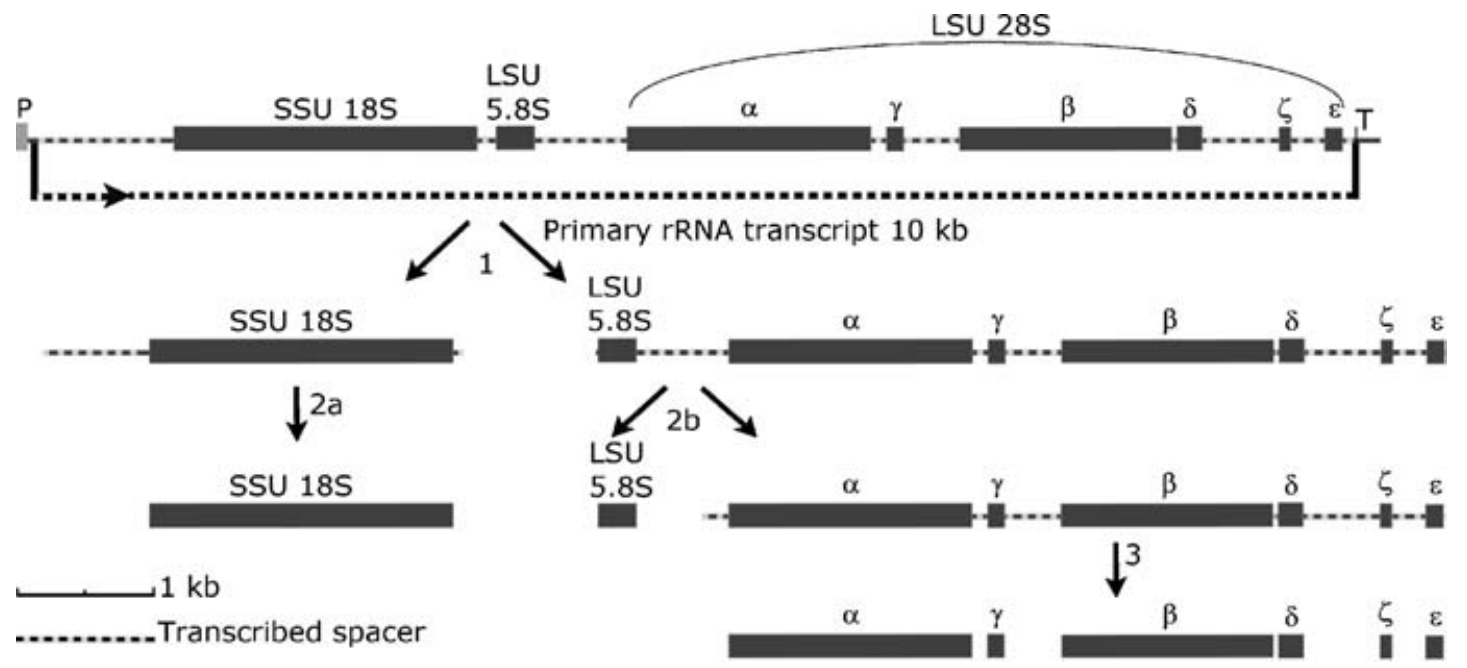

Fig. 1. Schematic representation of rDNA locus in Leishmania (based on L. major Acc. no. AC005806) and hypothetical processing pathway of Leishmania rRNA transcript. The initial steps take place in the nucleolus, where RNA polymerase I transcribes a primary rRNA transcript of $10 \mathrm{~kb}$ containing both external and internal transcribed spacers (1). Rapid processing at $5^{\prime}$ and $3^{\prime}$ of the primary transcript followed by cleavage results in pre-18S and pre-5.8S/28S. (2a) The pre-18S transcript is subsequently transported to the cytosol and further trimmed resulting in the mature $18 \mathrm{~S}$. (2b) Cleavage resulting in a pre-5.8S and pre-28S transcript; further maturation of $5.8 \mathrm{~S}$.

(3) Processing of pre-28S transcript resulting in the 6 characteristic fragments $\alpha, \beta, \gamma, \delta, \zeta$, and $\varepsilon$ rRNA. $(\mathrm{P}=$ promoter, $\mathrm{T}=$ terminator, $\mathrm{LSU}=$ large subunit, $\mathrm{SSU}=$ small subunit. $)$

monocistronic mature transcripts as in eukaryotes. Processing into single-gene units involves a combination of $5^{\prime}$ transsplicing and $3^{\prime}$ polyadenylation (LeBowitz et al. 1993). A second unique feature of kinetoplastids lies in the architecture of the cytosolic ribosomes. Processing of the primary rRNA transcript, also transcribed by an RNA polymerase I, results in mature $18 \mathrm{~S}$ SSU rRNA, 5.8S LSU rRNA and 6 discrete LSU rRNA species (referred to as $28 \mathrm{~S}$ $\alpha, \beta, \gamma, \delta, \zeta, \varepsilon$ in Leishmania and LSU1, LSU2, SR1, SR2, SR4, SR6 in Trypanosoma) that together represent the homolog of the $28 \mathrm{~S}$ LSU rRNA of other eukaryotes (White, Rudenko and Borst, 1986; Spencer et al. 1987; Hernandez, Diaz-de Leon and Castaneda, 1988; Martinez-Calvillo et al. 2001; Jensen et al. 2005) (Fig. 1).

In this paper we report another remarkable feature of RNA metabolism in the kinetoplastid Leishmania. Initially we were looking for differences in gene expression in a natural pentavalent antimonial $(\mathrm{Sb}(\mathrm{V}))$ resistant and sensitive strain by comparing oligo(dT) generated cDNA using suppression subtractive hybridisation (Diatchenko et al. 1996). Upon completion of screening, we were surprised to find that a substantial number of our candidate differentially expressed genes represent fragments of the $28 \mathrm{~S} \mathrm{LSU}$, which implies that rRNA in Leishmania can be polyadenylated. Since it was recently demonstrated that some mature rRNA transcripts can be polyadenylated in the eukaryotes Saccharomyces cerevisiae and Candida albicans (Fleischmann and Liu, 2001; Fleischmann, Liu and Wu, 2004; Kuai et al. 2004), we wanted to verify in detail whether Leishmania $28 \mathrm{~S}$ LSU fragments also have $3^{\prime}$ poly(A)-tails and to what extent this phenomenon occurs. Our results clearly demonstrate that a substantial part of precursor $28 \mathrm{~S}$ $\varepsilon$ rRNA undergoes extensive post-transcriptional polyadenylation. We found that the degree of this rRNA polyadenylation can vary between (i) different strains and (ii) the two different life-stages, the extracellular motile vector-life promastigote stage and the obligate intracellular amastigote mammalian stage.

\section{MATERIALS AND METHODS}

Leishmania strains and in vitro $S b(V)$ susceptibility testing

Strains. The L. braziliensis strains PER005, PER006 were directly isolated from skin biopsies from confirmed cutaneous leishmaniasis patients recruited at Instituto 'Alexander von Humbolt', Lima, Peru. The L. donovani strains BPK087/0, BPK091/0, BPK190/0 were isolated from bonemarrow aspirates from confirmed visceral leishmaniasis patients recruited at the BP Koirala Institute of Health Sciences, Dharan, Nepal. Promastigotes were grown in Tobie's blood agar medium at $26^{\circ} \mathrm{C}$ (Tobie, Von Brand and Mehlman, 1950) and harvested when log-phase was reached. Leishmania species identification was done by PCR-RFLP analysis of cysteine proteinase b (cpb) and gp63 genes for Nepalese and Peruvian strains, respectively. Briefly, DNA was extracted with the QIAmp DNA mini Kit (Qiagen), PCR amplified and digested as reported elsewhere (Victoir et al. 2003; Tintaya et al. 2004). Restriction patterns were resolved using the DNA Labchip kit on the 
Bioanalyzer 2100 (Agilent Technologies) and compared to patterns of reference strains.

In vitro amastigote generation. Starch-induced murine peritoneal macrophages were infected with late-stage promastigotes for $48 \mathrm{~h}$. The promastigotes were from the same passage as the concurrent in vitro tests. The adherent, infected macrophages were then washed with ice-cold PBS to remove extracellular promastigotes. Three $\mathrm{ml}$ of $0 \cdot 0125 \% \mathrm{SDS} / \mathrm{PBS}$ were added to each flask of infected macrophages, and gently agitated until the macrophages lifted and started to disintegrate. After dilution with PBS and mixing, the contents of each flask was aspirated through a $25 \mathrm{G} \frac{5}{8}$ needle, causing further shearing of the macrophages, and transferred to a $50 \mathrm{ml}$ centrifuge tube for final washing and centrifugation steps.

In vitro testing. Briefly, promastigotes were maintained in M199 medium supplemented with 20\% heat-inactivated foetal calf serum, at $25{ }^{\circ} \mathrm{C}$. Parasites were passaged weekly with promastigotes used at day 7 post-passage. The passage number was below 7 from the point of isolation. Starch-induced murine peritoneal macrophages were infected with promastigotes at a ratio of $7: 1 . L$. $(V$.$) braziliensis-infected$ cells were placed at $34{ }^{\circ} \mathrm{C}, 5 \% \mathrm{CO}_{2} /$ air mix, $L$. (L.) donovani-infected cells were maintained at $37{ }^{\circ} \mathrm{C}, 5 \%$ $\mathrm{CO}_{2}$ /air mix, for $24 \mathrm{~h}$. Level of infection was ascertained and infected macrophages were drugged with sodium stibogluconate $(\mathrm{Sb}(\mathrm{V}), \mathrm{GSK}, \mathrm{UK})$ for a total of $120 \mathrm{~h}$. The percentage inhibition and $\mathrm{IC}_{50}$ of the drugs were evaluated. Strains PER005 and BPK091/0 were characterized as $\mathrm{Sb}(\mathrm{V})$-sensitive and strains PER006,BPK190/0 and BPK087/0 as $\mathrm{Sb}(\mathrm{V})$-resistant.

\section{$R N A$ isolation and analysis}

All parasites cultured for RNA extraction were immediately upon harvest resuspended and disrupted in RNAqueous Lysis/Binding solution (Ambion) containing the protein denaturant guanidinium isothiocyanate which inhibits all enzymatic activity and as such protects the RNA. The resulting cell lysates were immediately frozen down at $-80{ }^{\circ} \mathrm{C}$ until RNA extraction. Total RNA was extracted using the RNAqueous kit (Ambion) and all samples were DNase-treated using the DNA-free kit (Ambion) to remove possible contaminating genomic DNA. Quality and quantity of the resulting RNA was determined using the RNA 6000 Nano Labchip kit on the Bioanalyzer 2100 (Agilent Technologies).

\section{Suppression Subtractive Hybridisation (SSH), differential screening and analysis of subtracted libraries}

The SMART PCR cDNA Synthesis kit (BD) was used to reverse transcribe total RNA from PER005 and PER006 amastigotes with a modified 30mer oligo(dT). SSH was performed to identify cDNAs specific for $\mathrm{Sb}(\mathrm{V})$-resistant L. braziliensis (PER006) using the PCR-Select cDNA Subtraction kit (BD). The resulting enriched cDNAs for the $\mathrm{Sb}(\mathrm{V})$ resistant strain were cloned into the plasmid pCR4TOPO using TOPO TA Cloning kit for Sequencing (Invitrogen). The reciprocal subtractive library was made in parallel to enrich for cDNAs specific for $\mathrm{Sb}(\mathrm{V})$-sensitive L. braziliensis (PER005). The obtained subtracted libraries were subsequently submitted to a differential screening step to eliminate false positive results, i.e. cDNAs that are common to both the $\mathrm{Sb}(\mathrm{V})$-resistant and $\mathrm{Sb}(\mathrm{V})$-sensitive strain, using the PCR Select Differential Screening kit (BD). All protocols were performed according to the manufacturer's instructions.

The selected cDNAs of the ' $\mathrm{Sb}(\mathrm{V})$-resistant library' were sequenced by the Flemish Institute for Biotechnology. Sequences were compared to public databases GenBank, using the Basic Local Alignment Search Tool (BLASTN) program from the National Centre for Biotechnology Information, and the Parasite Genomes WU-Blast2 kinetoplastids database administered by the European Bioinformatics Institute.

\section{Polyadenylation test}

Total RNA was reverse transcribed using a tailed oligo(dT) primer 5' GGTGAGCCCGCGTCACGG(T) $)_{12} 3^{\prime}$ with the Superscript III First Strand Synthesis System for RT-PCR (Invitrogen) using conditions advised by the manufacturer. The resulting ss cDNA was 10 times diluted and amplified by PCR applying conditions described elsewhere (Salles, Richards and Strickland, 1999), using the tailed oligo(dT) as reverse primer; and as forward primer 5' ACTGTACGGAACTCCTCGCCGGAATG $3^{\prime}$ for L. braziliensis and 5' GCTCTGGAAGCTGACAGTACGAACTCCTC $3^{\prime}$ for $L$. donovani, which are positioned in the ITS $5^{\prime}$ of the region coding for $28 \mathrm{~S} \varepsilon \mathrm{rRNA}$. The resulting products had a minimum length of $287 \mathrm{bp}$ and $271 \mathrm{bp}$, respectively, and were subsequently digested with an endonuclease. HinfI (New England Biolabs) was used for L. braziliensis (47 bp $+\min .240 \mathrm{bp}$ ) and MscI (New England Biolabs) was used for L. donovani $(84 \mathrm{bp}+\min .187 \mathrm{bp})$. PCR-products and digests were analysed using the DNA 1000 Labchip kit on the Bioanalyzer 2100 (Agilent Technologies).

PAT-products were cloned into pCR4-TOPO and 10 random clones were sequenced as described before.

\section{Real-Time Quantitative PCR}

Total RNA ( $\pm 100 \mathrm{ng} / \mathrm{rxn})$ was reverse transcribed at $55{ }^{\circ} \mathrm{C}$ with Transcriptor Reverse Transcriptase 
(Roche) using conditions recommended by the manufacturer with either a $15 \mathrm{mer}$ oligo $(\mathrm{dT})$ primer for specific transcription of poly(A)-RNA or the primer 'EPSREV' 5' CGAGCCCCACCGAAGTG $3^{\prime}$ for specific transcription of $\varepsilon$ rRNA. The resulting cDNA was 10 times diluted and $1 \mu \mathrm{l}$ was added to $25 \mu \mathrm{l}$ quantitative $\mathrm{PCR}$ reactions, which contained $1 \times$ iQ Sybr Green Supermix (Bio-Rad) and $100 \mathrm{~nm}$ of both forward primer (5' TGGAATACACCAAACCACAACCT 3 ' for $L$. braziliensis and 5' CGGAATACACCAAACAACAACAT $3^{\prime}$ for $L$. donovani, positioned in ITS $5^{\prime}$ of $\varepsilon$ fragment) and reverse primer (EPSREV). All quantitative assays were done on the iCycler (Bio-Rad) using the following thermal profile: initial denaturation at $95{ }^{\circ} \mathrm{C}$ for $5 \mathrm{~min}$ followed by 30 cycles with denaturation at $95{ }^{\circ} \mathrm{C}$ for $30 \mathrm{sec}$, annealing at $60{ }^{\circ} \mathrm{C}$ for $15 \mathrm{sec}$ and extension at $72{ }^{\circ} \mathrm{C}$ for $15 \mathrm{sec}$. All reactions were done in triplicate, with their mean $\mathrm{Ct}$ used for data analysis.

\section{Polyadenylation Value (PAV), Differential Polyadenylation Factor (DPF) and applied statistics}

Based on the mathematical equation describing the exponential nature of PCR-amplification, the ratio of the starting amount of polyadenylated precursor $\varepsilon \operatorname{rRNA}\left(\varepsilon_{\mathrm{A}}\right)$ versus the starting amount of total precursor $\varepsilon$ rRNA $\left(\varepsilon_{\mathrm{T}}\right)$, here defined as 'polyadenylation value (PAV)', can be written as:

$\mathrm{PAV}=\varepsilon_{\mathrm{A}} / \varepsilon_{\mathrm{T}}=\mathrm{Eff}^{(\mathrm{Ct}, \mathrm{T}-\mathrm{Ct}, \mathrm{A})}$

where $\mathrm{Ct}, \mathrm{T}$ and $\mathrm{Ct}, \mathrm{A}$ equal the threshold cycle values in the quantitative PCR on $\varepsilon$ specific-primed cDNA and oligo(dT)-primed cDNA respectively. Eff equals the PCR-efficiency, and was determined based on 4-point 4-fold serial dilution standard curves. PAV only reflects an estimation of the fraction of precursor $\varepsilon$ rRNA that is polyadenylated, since $\mathrm{Ct}, \mathrm{T}$ and $\mathrm{Ct}, \mathrm{A}$ are measured on 2 differently primed sources of cDNA (and no guarantee can be obtained for measuring equal PCR threshold cycle values for equal transcript numbers). Comparison between PAVs of 2 samples assesses whether $\varepsilon$-polyadenylation occurs to a different degree in these samples, and is here defined as the 'differential polyadenylation factor' DPF of sample A versus sample B with:

$\mathrm{DPF}=\mathrm{PAV}$ sample $\mathrm{A} / \mathrm{PAV}$ sample $\mathrm{B}$

where sample A and sample B can represent (i) promastigote and amastigote of an individual strain or (ii) 2 different strains (in the same life-stage) belonging to the same species. Important to note is that by comparing 2 samples, the putative bias introduced by the 2 different RT priming methods is completely neutralized. The significance of the DPF is determined using $95 \%$ confidence intervals (CI), based on 5 repeated quantitative $\mathrm{PCR}$ runs on the same batch

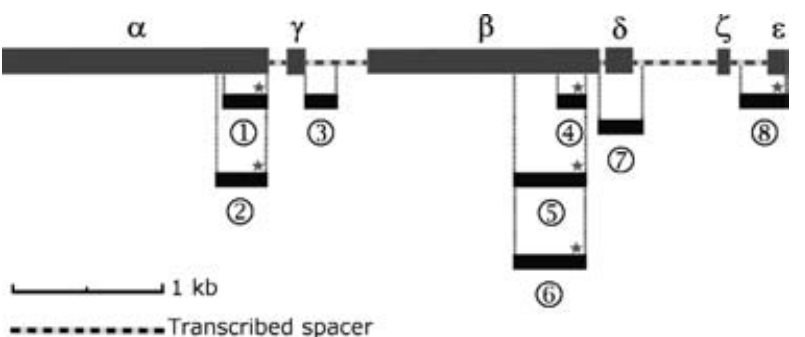

Fig. 2. SSH-results: Alignment of $8 \mathrm{cDNAs}$ from the ' $\mathrm{Sb}(\mathrm{V})$ resistant library' with the rDNA locus of Leishmania major (Acc no. AC005806). cDNAs representing the polyadenylated 3 ' ends of the original RNA as suggested by the presence of the tailed oligo(dT)-primer used during SSH cDNA synthesis are indicated by an asterisk. cDNAs $3 \& 7$ have a RsaI - site at their $3^{\prime}$, which result from the SSH's RsaI restrictionstep performed immediately after cDNA-synthesis.

However, since cDNA synthesis was primed by oligo(dT) it is very likely that these restriction fragments were originally part of a polyadenylated precursor rRNA.

of $\varepsilon$ specific-primed and oligo(dT)-primed cDNA unless otherwise mentioned. $95 \%$ CI were calculated on $\log$ transformed DPF values, using the T.INV function in MS Excel to calculate the critical $t$ value $[\mathrm{CI}=$ mean DPF \pm S.E.M. $* t$, with S.E.M. $=$ standard error of the mean, and $t=$ critical $t$ value $=$ T.INV(0·05, degrees of freedom)] (Motulsky, 1995).

\section{RESULTS}

\section{Enrichment for polyadenylated $r R N A$ by $S S H$}

The ' $\mathrm{Sb}(\mathrm{V})$ resistant library' contained 11 cDNAs characteristic for $\mathrm{Sb}(\mathrm{V})$ resistant L. braziliensis. Sequence analysis clearly identified 8 of these cDNAs as parts of the $28 \mathrm{~S} \mathrm{LSU} \mathrm{rRNA} \mathrm{homologue} \mathrm{(Fig.} \mathrm{2);}$ the other 3 sequences correspond to protein-coding genes and will be reported elsewhere. The predominant presence of $28 \mathrm{~S}$ rRNA fragments in a 30 mer oligo(d'T) primed cDNA library was unexpected, as this suggests that the original $28 \mathrm{~S}$ rRNA fragments had a poly(A)-tail at their $3^{\prime}$ end which is generally assumed to be a hallmark of mRNA. Remarkably, the 6 cDNAs that represent the polyadenylated $3^{\prime}$ ends of the original RNA as suggested by the presence of the oligo(dT) primer, all align approximately to the mature 3 ' end of one of the 28S LSU small fragments (Fig. 2). This implies that these fragments were polyadenylated after undergoing the primary rRNA processing steps that created the $3^{\prime}$ mature ends. Furthermore, cDNAs 3, 7 and 8 contain transcribed spacers, which suggests that these $\mathrm{cDNAs}$ originate form incompletely processed rRNA or precursor rRNA (Fig. 2). Together, these findings suggest that rRNA in Leishmania can undergo polyadenylation during processing of the primary rRNA transcript. In order to further explore this hypothesis, we focused on cDNA 8 as this was the only sequence 

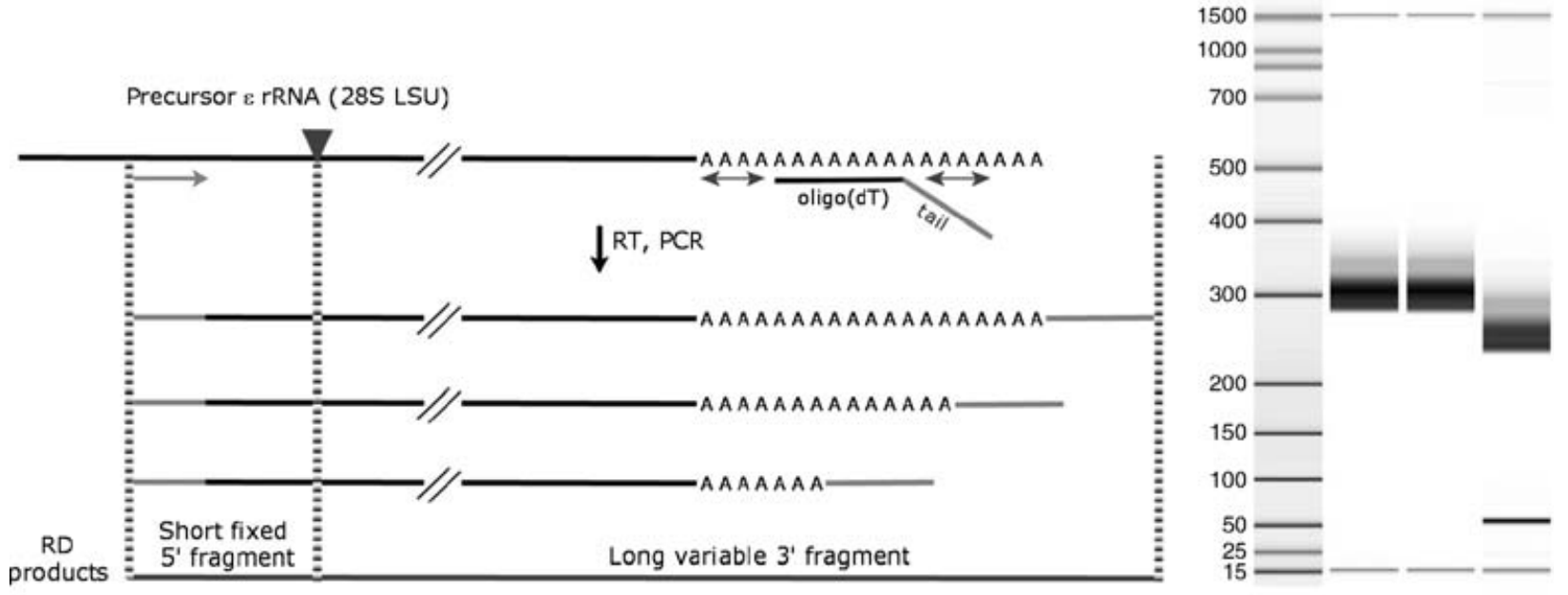

A

\section{B}

Fig. 3. (A) Schematic representation of PAT design for Leishmania braziliensis and L. donovani including 3 steps (i) RT with tailed oligo(dT)-primer, (ii) PCR with rRNA specific forward primer and tailed oligo(dT)-primer yielding a mixture of PCR-products representing the total length of the poly(A) tail and (iii) restriction digest (RD) to confirm $3^{\prime}$ variability (restriction site $=$ triangle). (B) Banding pattern of PAT on $\varepsilon$ rRNA in L. braziliensis, demonstrating the presence of a poly(A)-tail of \pm 100 A's at the $3^{\prime}$ end of the rRNA transcript with lane 1 DNA ladder, lane 2 amastigotes PER005, lane 3 amastigotes PER006, lane 4 amastigotes PER005 digested with HinfI.

available that contained both transcribed spacer (marker of precursor rRNA) and mature 3' end of a $28 \mathrm{~S}$ rRNA fragment (possible polyadenylation site).

\section{Polyadenylation of precursor $\varepsilon r R N A$ in}

$\mathrm{L}$. braziliensis and L. donovani

A polyadenylation test (PAT) was developed to check the presence and length of poly(A)-tails in precursor $\varepsilon$ rRNA. The first step involved reverse transcription of total RNA using a 12-mer oligo(dT)-primer with a GC-rich tail at its $5^{\prime}$ end. This RT step synthesizes a heterogeneous pool of ss cDNA that has been primed at all possible positions along the poly(A)-tail at the $3^{\prime}$ end of RNA. If precursor $\varepsilon$ rRNA is indeed polyadenylated, then the subsequent PCR amplification using a primer specific for the $5^{\prime}$ ITS of the $\varepsilon$ fragment and the tailed 12-mer oligo(d'T)-primer should yield a mixture of PCR-products representing the length of the poly(A)-tail (Fig. 3A).

We tested both sensitive and resistant $L$. braziliensis strains (PER005 and PER006) and clearly observed PCR-products with a $100 \mathrm{bp}$ variation in length in both strains, suggesting that precursor $\varepsilon$ rRNA has a poly(A)-tail of approximately $100 \mathrm{bp}$ (Fig. 3B). These poly(A)-tails are not the result of mispriming in A-rich regions of contaminating genomic DNA as (i) negative RT controls (i.e. without transcriptase) had negative PAT results and (ii) PAT using genomic DNA as template was negative (data not shown). To confirm that the variation in length is not due to false priming at the $5^{\prime}$ end of the product, PAT-products were digested with HinfI. This enzyme should cleave $49 \mathrm{bp}$ downstream of the $5^{\prime}$ end, resulting in a constant $5^{\prime}$ short fragment and a heterogeneous 3' long fragment (Fig. 3A). Restriction analysis of the PAT-products of PER005 and PER006 confirmed indeed that the $3^{\prime}$ end is the cause of the heterogeneous length (Fig. 3B).

To further demonstrate polyadenylation of precursor $\varepsilon$ rRNA, we cloned the PER006 PATproducts into a plasmid and sequenced the inserts of 10 random clones. This analysis identified all inserts as precursor $\varepsilon$ rRNA with a $3^{\prime}$ poly(A) tail varying in length between 15 and $107 \mathrm{bp}$ (Fig. 4). Furthermore, in all cases the poly(A)-tail was positioned at the $3^{\prime}$ mature end of $\varepsilon$ rRNA, a finding which was also observed in the cDNAs of the subtracted library.

As polyadenylation of precursor $\varepsilon$ rRNA was observed in 2 different strains of L. braziliensis, we debated whether this is a widespread phenomenon in Leishmania species. Therefore, an analogous PAT was designed for the Old World species L. donovani. The L. donovani strains BPK087/0 and BPK190/0 both presented similar profiles of PAT-products and restriction digests as the L. braziliensis strains (data not shown).

\section{Quantification of the polyadenylated fraction of total precursor $\varepsilon$ rRNA in $\mathrm{L}$. braziliensis \\ and $\mathrm{L}$. donovani}

SSH indicated that polyadenylated precursor $\varepsilon$ rRNA distinguishes PER006 amastigotes from PER005 amastigotes. However, PAT showed that polyadenylation of precursor $\varepsilon$ rRNA occurs in 
PERO 06 C1 1 GGGGCATTTACGTCCCGAGGCGCTGAACCTTGAGGCCTGAAATTTCATGCTCAGGGACA AAAAAAAAAAAAAAAAAAAAAA............ PERO0 6 Cl 2

PERO0 6 C1 3 PERO06 C1 4 PERO 06 C1 4 PERO06 C1 6 PERO06 C1 7 PERO06 C1 7 PERO06 Cl 7 PERO 06 C1 7 3'of E(L.major)
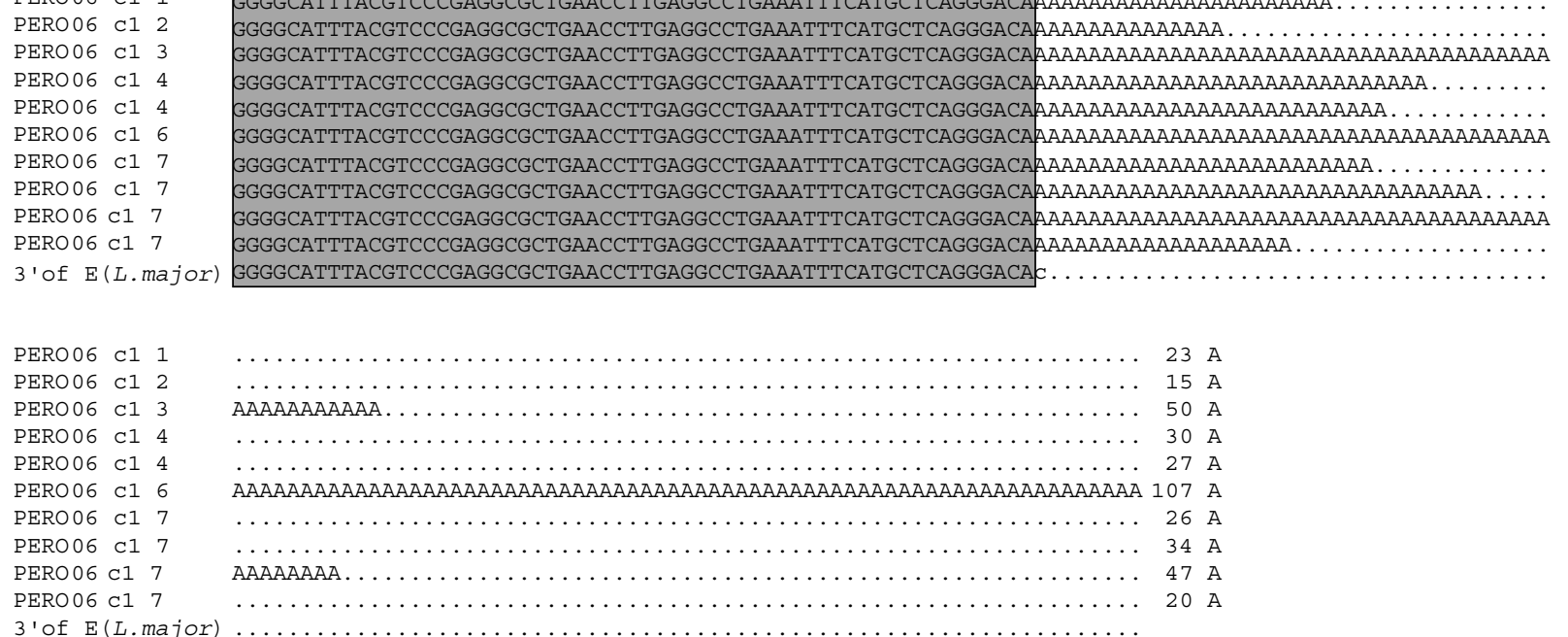

Fig. 4. Alignment of $3^{\prime}$ end sequences of 10 cloned $\varepsilon$ PAT-PCR products of PER006 with $3^{\prime}$ end of $\varepsilon$ rRNA of Leishmania major (AC005806), demonstrating the presence of a poly(A)-tail at the predicted end of the $\varepsilon$ rRNA varying in length between $15 \mathrm{bp}$ and $107 \mathrm{bp}$. The complete sequence of the cloned L. braziliensis $\varepsilon$ rRNA is available in GenBank data bank under Accession no. AY730681.

amastigotes of both strains. Consequently, we suspected that there might be a quantitative difference in polyadenylated precursor $\varepsilon$ rRNA in these 2 strains. Assuming that not all precursor $\varepsilon$ rRNA is polyadenylated, we hypothesized that the ratio of the amount of polyadenylated precursor $\varepsilon$ rRNA versus the amount of total precursor $\varepsilon$ rRNA, further referred to as the 'polyadenylation value' $(\mathrm{PAV})$, varies in different strains.

A real-time quantitative PCR was developed to verify this hypothesis in L. braziliensis and L. donovani. This PCR allowed quantification in a particular sample of both (i) polyadenylated precursor $\varepsilon$ rRNA by using oligo(dT) primed cDNA and (ii) all precursor $\varepsilon$ rRNA by using $\varepsilon$-specific primed cDNA. The quantitative data from these 2 PCRs were used to determine the PAV of that sample (see Materials and Methods section). PAVs allowed us to compare the degree of precursor $\varepsilon$ rRNA polyadenylation between different samples by calculating the 'differential polyadenylation factor' (DPF, see Materials and Methods section).

The DPF of PER006 amastigotes versus PER005 amastigotes was $3 \cdot 28$ with $95 \%$ CI [ $3 \cdot 05,3 \cdot 52]$, which confirms the $\mathrm{SHH}$ results and more specifically shows that the polyadenylated fraction of precursor $\varepsilon$ rRNA in PER006 amastigotes is at least 3 times larger than in PER005 amastigotes, with 95\% confidence (Fig. 5A). The same experiment was repeated for a similar couple of L. donovani strains, BPK190/0 and BPK091/0, and demonstrated that amastigotes of different $L$. donovani strains can also have differential polyadenylation of precursor $\varepsilon$ rRNA (Fig. 5A). To verify whether this differential polyadenylation also occurs in the promastigote stage, we determined the corresponding promastigote DPFs. Surprisingly, for both couples, the DPF is approximately 1,

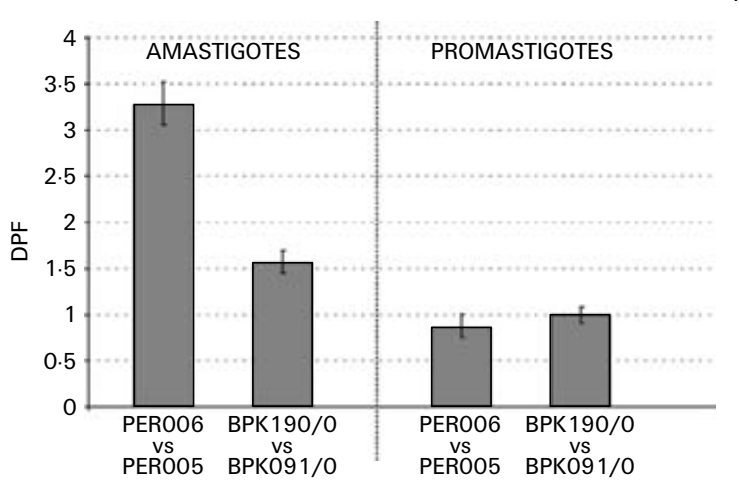

B

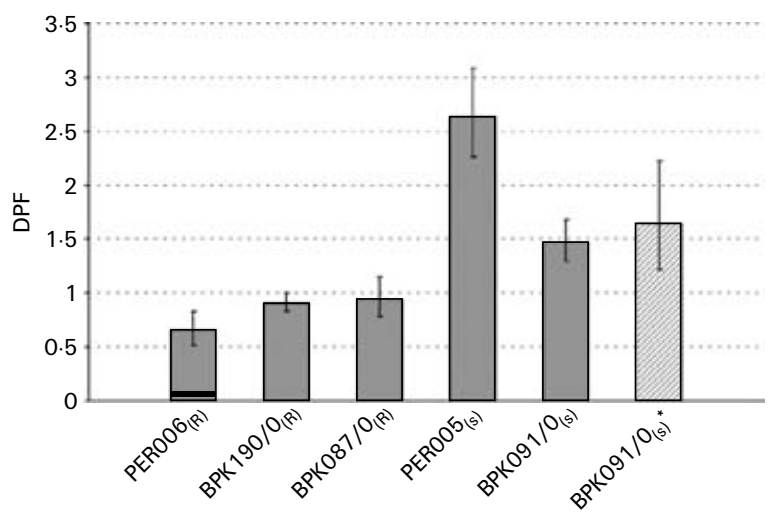

Fig. 5. (A) DPFs comparing different strains (in the same life-stage, indicated on top) belonging to the same species with $95 \%$ CI. (B) DPF of promastigotes versus amastigotes for individual strains with $95 \% \mathrm{CI}$. The grey bars are based on data of 5 repeated quantitative PCRs; the hatched bar (BPK091/0*) is based on 3 completely repeated assays (i.e. cDNA synthesis and quantitative PCR) and demonstrates that the assay is reproducible, as explained in the text. De index in each strain's name refers to the in vitro $\mathrm{SbV}$ susceptibility profile of the strain, with $(\mathrm{R})=\mathrm{Sb}(\mathrm{V})$ resistant and $(\mathrm{S})=\mathrm{Sb}(\mathrm{V})$ sensitive. 
indicating a similar degree of precursor $\varepsilon$ rRNA polyadenylation in promastigotes of different strains belonging to the same species (Fig. 5A). Furthermore, based on the PAVs, we estimate that more than half of the precursor $\varepsilon$ rRNA can be polyadenylated in both promastigotes and amastigotes.

We also used the available PAVs to compare the degree of precursor $\varepsilon$ rRNA polyadenylation in the 2 life-stages of an individual strain; the corresponding DPFs for the strains PER006, PER005, BPK190/0, BPK091/0 and BPK087/0 are presented in Fig. 5B. The graph shows that in an individual strain the polyadenylated fraction of precursor $\varepsilon$ rRNA in promastigotes can be (i) smaller than in amastigotes (PER006, BPK190/0), (ii) approximately equal to amastigotes (BPK087/0) or (iii) larger than in amastigotes (PER005, BPK091/0). Interestingly, the 3 strains with a $\mathrm{DPF} \leqslant 1$ are $\mathrm{Sb}(\mathrm{V})$ resistant, while the 2 strains with a $\mathrm{DPF}>1$ are $\mathrm{Sb}(\mathrm{V})$ sensitive.

The $95 \%$ CI of the reported DPFs reflect the variation in 5 repeated quantitative PCR experiments using the same batch of cDNA (see experimental procedures). We also wanted to assess the reproducibility of the complete assay (including the RT-step). Therefore, we determined the DPF and its $95 \%$ CI a second time for 1 strain, BPK091/0, but this time based on the data collected from 3 repeated experiments that included cDNA-synthesis with oligo(dT) primer and $\varepsilon$-specific primer and the concomitant quantitative PCRs. Overall, a similar result was obtained as in the previous experiment, except that the $95 \% \mathrm{CI}$ is wider (Fig. 5B), which is in perfect agreement with the use of a higher critical $t$-value for the calculation of $95 \%$ CI (3 vs 5 repeated measurements) and with additional variation introduced by the inclusion of cDNA synthesis in the repeated experiments.

\section{I S CUSSION}

We have demonstrated in Leishmania braziliensis and $L$. donovani that precursor $\varepsilon$ rRNA can have poly(A)tails as long as $100 \mathrm{bp}$, which clearly shows that the $\varepsilon$ rRNA fragment of the ribosome's LSU can be polyadenylated before completion of post-transcriptional maturation. Furthermore, according to the quantitative data, it is estimated that more than half of precursor $\varepsilon$ rRNA can be polyadenylated. The sequencing results showed that the poly(A)-tail of the precursor $\varepsilon$ fragment is located at the mature $3^{\prime}$ end of $\varepsilon$, which further indicates that the polyadenylation occurs during maturation, more specifically after cleavage of the $3^{\prime}$ ETS and is not coupled to transcription termination as observed with mRNA. The subtractive cDNA cloning suggests that other precursor LSU fragments can be polyadenylated as well, but further experiments are required to confirm these findings. We do not exclude the possibility that some of the detected polyadenylated precursor
rRNAs are an artefact caused by RNA manipulation. It has been demonstrated that minimal enzymatic RNA end-labelling occurs in homogenized cells. (White and Borst, 1987) However, we are confident that this spurious enzymatic activity, if any, could only have had a minor contribution to the extensive rRNA polyadenylation described here as (i) all enzymatic activity was inhibited during cell disruption and (ii) major modifications, such as addition of $100 \mathrm{bp}$ polyA-tails, are unlikely to be effected by transient exposure to cellular enzymes.

Considering the evolutionary origin of Leishmania, a primordial eukaryote, we reviewed the literature describing polyadenylation of RNA in both prokaryotes and eukaryotes to find explanations for this surprising phenomenon. In prokaryotes, polyadenylation of rRNA has been reported in the context of degradation. Since the presence of the poly(A)tail disturbs the secondary structure at the $3^{\prime}$ end of rRNA, it makes the $3^{\prime}$ end accessible to degrading exonucleases (Carpousis, Vanzo and Raynal, 1999). Consequently, it has been hypothesized that poly(A)-tails mark prokaryotic ribosomal RNA for degradation and are, as such, part of a quality control mechanism active during rRNA formation/ maturation. This mechanism enables the cell to eliminate badly processed rRNA that would otherwise result in non-functional ribosomes ( $\mathrm{Li}$, Pandit and Deutscher, 1998; Deutscher, 2003). Recent studies on Saccharomyces cerevisiae and Candida albicans suggest that an analogous quality control mechanism through polyadenylation might also be employed by eukaryotes to degrade badly processed rRNA and tRNA (Fleischmann and Liu, 2001; Fleischmann, Liu and $\mathrm{Wu}, 2004$; Kadaba et al. 2004; Kuai et al. 2004). However, here for Leishmania, the degradation hypothesis is less convincing as the quantitative results indicate that more than half of precursor ribosomal RNA can be polyadenylated. Consequently, if ribosomal poly(A)-tails indeed reflect a degradation mechanism, then ribosomal processing would be very inefficient and uneconomic. Alternative functional roles of ribosomal poly(A)tails have not been described yet. However, the various processing steps occurring during the maturation of the kinetoplastid primary ribosomal transcript have not been characterized completely. It will be interesting to see if the $3^{\prime}$ end maturation of the rRNA LSU fragments is in any way related to the cleavage/polyadenylation mechanism effecting the $3^{\prime}$ end formation of mRNA during the processing of the polycistronic pre-mRNAs in kinetoplastids.

We further characterized the described rRNA polyadenylation phenomenon in Leishmania using a comparative quantitative method. It was clearly shown that the degree of polyadenylation of precursor $\varepsilon$ rRNA is variable in different strains and in different life-stages of a strain. This variation is unlikely to be associated with the growth phase of 
the specific parasite as all studied parasites were consistently harvested at the same growth phase. It is interesting to point out that the polyadenylation phenomenon is present in 2 distant evolutionary species, L. braziliensis and L. donovani, indicating that this is probably a conserved feature of Leishmania. The differential polyadenylation between promastigotes and amastigotes of a particular strain agrees with the different biological nature of the 2 life-stages. Promastigotes reside in the midgut of the sandfly and amastigotes in mammalian macrophages. Both life-stages are specifically adapted to their respective environments and this is reflected in numerous physiological and biochemical differences (McConville and Ralton, 1997; Mazareb, Fu and Zilberstein, 1999; Burchmore and Barrett, 2001). The described differential polyadenylation is, to our knowledge, the first reported difference on the level of non-translated RNA. Comparing different strains of the same species, we noticed that the degree of polyadenylation is similar in promastigotes but varies in amastigotes, resulting in varying DPFs (promastigotes $v s$ amastigotes) of individual strains. Interestingly, we noticed a possible association between the individual strain's DPF and its in vitro $\mathrm{SbV}$ susceptibility in amastigote form. Whether the differential polyadenylation factor of an individual strain is indeed a direct or indirect marker for a strain's $\mathrm{Sb}(\mathrm{V})$ susceptibility, needs to be further explored and confirmed in future studies.

The demonstration of polyadenylation of rRNA conflicts with the traditional view that poly(A)-tails are a distinctive feature of $\mathrm{mRNA}$. As the techniques utilised to study mRNA are often based on that feature, future research should take into account the possibility that a high background can be present due to polyadenylated rRNA. More specifically in our study, it is very likely that differentially expressed mRNA transcripts in $\mathrm{Sb}(\mathrm{V})$ sensitive and resistant Leishmania could not be detected due to the overwhelming presence of polyadenylated precursor rRNA.

We thank Dr Simon Croft and Dr Jan Van den Abbeele for critically reading the manuscript. This work was supported by the Fifth Framework Programme of the European Commission Community Research (Contract: ICA-CT2001-10076).

\section{REFERENCES}

Burchmore, R. J. and Barrett, M. P. (2001). Life in vacuoles-nutrient acquisition by Leishmania amastigotes. International journal for Parasitology 31, 1311-1320.

Carpousis, A. J., Vanzo, N. F. and Raynal, L. C. (1999). mRNA degradation. A tale of poly(A) and multiprotein machines. Trends in Genetics 15, 24-28.

Colgan, D. F. and Manley, J. L. (1997). Mechanism and regulation of mRNA polyadenylation. Genes and Development 11, 2755-2766.
Deutscher, M. P. (2003). Degradation of stable RNA in bacteria. The Fournal of Biological Chemistry 278, 45041-45044.

Diatchenko, L., Lau, Y. F., Campbell, A. P., Chenchik, A., Moqadam, F., Huang, B., Lukyanov, S., Lukyanov, K., Gurskaya, N., Sverdlov, E. D. and Siebert, P. D. (1996). Suppression subtractive hybridization: a method for generating differentially regulated or tissuespecific cDNA probes and libraries. Proceedings of the National Academy of Sciences, USA 93, 6025-6030.

Fleischmann, J. and Liu, H. (2001). Polyadenylation of ribosomal RNA by Candida albicans. Gene 265, 71-76.

Fleischmann, J., Liu, H. and Wu, C. P. (2004). Polyadenylation of ribosomal RNA by Candida albicans also involves the small subunit. $B M C$. Molecular Biology 5, 17-22.

Fromont-Racine, M., Senger, B., Saveanu, C. and Fasiolo, F. (2003). Ribosome assembly in eukaryotes. Gene 313, 17-42.

Hernandez, R., Diaz-De Leon, F. and Castaneda, M. (1988). Molecular cloning and partial characterization of ribosomal RNA genes from Trypanosoma cruzi. Molecular and Biochemical Parasitology 27, 275-279.

Hernandez-Rivas, R., Martinez-Calvillo, S., Romero, M. and Hernandez, R. (1992). Trypanosoma cruzi 5S rRNA genes: molecular cloning, structure and chromosomal organization. FEMS Microbiology Letters 71, 63-67.

Hirose, Y. and Manley, J. L. (1998). RNA polymerase II is an essential mRNA polyadenylation factor. Nature, London 395, 93-96.

Jensen, B. C., Brekken, D. L., Randall, A. C., Kifer, C. T. and Parsons, M. (2005). Species specificity in ribosome biogenesis: a nonconserved phosphoprotein is required for formation of the large ribosomal subunit in Trypanosoma brucei. Eukaryotic Cell 4, $30-35$.

Kadaba, S., Krueger, A., Trice, T., Krecic, A. M., Hinnebusch, A. G. and Anderson, J. (2004). Nuclear surveillance and degradation of hypomodified initiator tRNAMet in S. cerevisiae. Genes and Development 18, 1227-1240.

Kuai, L., Fang, F., Butler, J. S. and Sherman, F. (2004). Polyadenylation of rRNA in Saccharomyces cerevisiae. Proceedings of the National Academy of Sciences, USA 101, 8581-8586.

Lebowitz, J. H., Smith, H. Q., Rusche, L. and Beverley, S. M. (1993). Coupling of poly(A) site selection and trans-splicing in Leishmania. Genes and Development 7, 996-1007.

Li, Z., Pandit, S. and Deutscher, M. P. (1998). Polyadenylation of stable RNA precursors in vivo. Proceedings of the National Academy of Sciences, USA 95, 12158-12162.

Martinez-Calvillo, S., Sunkin, S. M., Yan, S., Fox, M., Stuart, K. and Myler, P. J. (2001). Genomic organization and functional characterization of the Leishmania major Friedlin ribosomal RNA gene locus. Molecular and Biochemical Parasitology 116, 147-157.

Mazareb, S., Fu, Z. Y. and Zilberstein, D. (1999). Developmental regulation of proline transport in 
Leishmania donovani. Experimental Parasitology 91, 341-348.

McConville, M. J. and Ralton, J. E. (1997).

Developmentally regulated changes in the cell surface architecture of Leishmania parasites. Behring Institute Mitteilungen 99, 34-43.

Motulsky, H. (1995). Intuitive Biostatistics, Oxford University Press, New York.

Paule, M. R. and White, R. J. (2000). Survey and summary: transcription by RNA polymerases I and III. Nucleic Acids Research 28, 1283-1298.

Proudfoot, N. and O'Sullivan, J. (2002).

Polyadenylation: a tail of two complexes. Current Biology 12, R855-R857.

Reeder, R. H. (1990). rRNA synthesis in the nucleolus. Trends in Genetics 6, 390-395.

Salles, F. J., Richards, W. G. and Strickland, S. (1999). Assaying the polyadenylation state of mRNAs. Methods 17, 38-45.

Sogin, M. L., Gunderson, J. H., Elwood, H. J., Alonso, R. A. and Peattie, D. A. (1989).

Phylogenetic meaning of the kingdom concept: an unusual ribosomal RNA from Giardia lamblia. Science 243, 75-77.

Sollner-Webb, B. and Mougey, E. B. (1991). News from the nucleolus: rRNA gene expression. Trends in Biochemical Sciences 16, 58-62.

Spencer, D. F., Collings, J. C., Schnare, M. N. and Gray, M. W. (1987). Multiple Spacer sequences in the nuclear large subunit ribosomal RNA gene of Crithidia fasciculata. The EMBOF Fournal 6, 1063-1071.
Tintaya, K. W., Ying, X., Dedet, J. P., Rijal, S., De-Bolle, X. and Dujardin, J. C. (2004). Antigen genes for molecular epidemiology of leishmaniasis: polymorphism of cysteine proteinase B and surface metalloprotease glycoprotein 63 in the Leishmania donovani complex. Fournal of Infectious Diseases 189, 1035-1043.

Tobie, E. J., Von Brand, T. and Mehlman, B. (1950). Cultural and physiological observations on Trypanosoma rhodesiense and Trypanosoma gambiense. The Fournal of Parasitology 36, 48-54.

Victoir, K., De Doncker, S., Cabrera, L., Alvarez, E., Arevalo, J., Llanos-Cuentas, A., Le Ray, D. and Dujardin, J. C. (2003). Direct identification of Leishmania species in biopsies from patients with American tegumentary leishmaniasis. Transactions of the Royal Society of Tropical Medicine and Hygiene 97, 80-87.

White, T. C. and Borst, P. (1987). RNA end-labeling and RNA ligase activities can produce a circular $\mathrm{rNA}$ in whole cell extracts from trypanosomes. Nucleic Acids Research 15, 3275-3290.

White, T. C., Rudenko, G. and Borst, P. (1986). Three small RNAs within the $10 \mathrm{~kb}$ trypanosome rRNA transcription unit are analogous to domain VII of other eukaryotic 28S rRNAs. Nucleic Acids Research 14, 9471-9489.

Zhao, J., Hyman, L. and Moore, C. (1999). Formation of mRNA 3' ends in eukaryotes: mechanism, regulation, and interrelationships with other steps in mRNA synthesis. Microbiology and Molecular Biology Reviews 63, 405-445. 